\title{
Correction of Spectral Interference of Ethanol and Glucose for Rice Wine Evaluation
}

\author{
Satoru Suzuki, Satsuki Hosono, Pradeep K. W. Abeygunawardha, Ichiro Ishimaru, Kenji Wada, and \\ Akira Nishiyama
}

\begin{abstract}
Rice wine, the traditional Japanese alcohol, contains ethanol and glucose, whose concentrations are evaluated to manage their quality. Recently, infrared techniques have been introduced to rice wine measurement, and absorption spectra are analyzed to monitor rice wine components during fermentation. However, it is difficult to precisely evaluate ethanol and glucose concentrations because of an overlapping of their absorption peaks. This paper proposes a spectral separation method using a single absorption band for evaluating the ethanol and glucose concentrations in rice wine. We evaluate their concentrations by separating a mixed spectrum into ethanol and glucose spectra. The simulation results showed a decrease in the estimation errors of ethanol and glucose concentrations from $5.40 \pm 5.41 \%(\mathrm{v} / \mathrm{v})$ and $0.73 \pm 0.73 \mathrm{~g} / \mathrm{dl}$, respectively, using regression analysis (RA) to $0.46 \pm 0.65 \%(\mathrm{v} / \mathrm{v})$ and $0.40 \pm 0.57 \mathrm{~g} / \mathrm{dl}$, respectively, using the proposed method.
\end{abstract}

Index Terms-Ethanol andglucose evaluation, regression analysis, spectral separation.

\section{INTRODUCTION}

In rice wine fermentation, it is important to measure ethanol and glucose concentrations to manage the quality of products. Recently, near-infrared (NIR) and mid-infrared (MIR) spectroscopies have been introduced to evaluate the components in alcoholic beverages [1]-[3]. To evaluate components in an alcoholic beverage from its measured spectrum, chemometrics also plays an important role.

Chemometrics has been used for both quantitative and qualitative evaluation in NIR and MIR measurement spectra [4]-[10]. Multiple linear regression (MLR) has been used for soil analysis [4] and monitoring the stability of biodiesel [5]. MLR linearly relates a sample concentration to an absorption spectrum. Principal component regression (PCR) has been used for analyzing polyphenolic compounds of red wines [6] and olive oils [7]. PCR is a method that combines principal component analysis (PCA) and MLR. PCR can avoid

Manuscript received September 10, 2014; revised December 8, 2014 This work was supported by a Young Research Grant from Kagawa University in Japan.

Satoru Suzuki, Satsuki Hosono, Pradeep K. W. Abeygunawardha, and Ichiro Ishimaru are with the Department of Intelligent Mechanical Systems Engineering, Kagawa University, 2217-20 Hayashi-cho Takamatsu Kagawa Japan (e-mail: s-suzuki@eng.kagawa-u.ac.jp, s11t446@stmail.eng.kagawa-u.ac.jp, kumara@eng.kagawa-u.ac.jp, ishimaru@eng.kagawa-u.ac.jp).

Kenji Wada is with the Department of Chemistry for Medicine, Kagawa University, 1750-1 Ikenobe Miki-cho Kita-gun Takamatsu Kagawa Japan (e-mail:wadaken@med.kagawa-u.ac.jp).

Akira Nishiyama is with the Department of Pharmacology, Kagawa University, 1750-1 Ikenobe Miki-cho Kita-gun Takamatsu Kagawa Japan (e-mail: akira@med.kagawa-u.ac.jp). multi-colinearity, a cause of performance degradation in MLR, by converting input variables into non-correlated variables. Moreover, PCA can reduce noise in the data, thus the $\mathrm{S} / \mathrm{N}$ ratio may be improved. However, PCR does not guarantee the correlation between converted non-correlated variables and a sample concentration. Partial least square regression (PLSR) solves this problem and achieves better performance compared to PCR. PLSR has been used to evaluate glucose, fructose, ethanol and glycerol to monitor red wine fermentation [8], and to determine eleven parameters in rice wine: total sugar, non-sugar solid, glucose, isomaltose, isomaltotriose, maltose, panose, total acid, amid acid nitrogen, $\mathrm{pH}$ and lactic acid [9]. To improve the estimation performance, PLSR has been combined with wavenumber selection [10]. However, we cannot expect the reliability of PLSR with wavenumber selection when absorption peaks of multiple components overlap.

As an approach to evaluate multiple components in the spectrum of a mixture, signal separation has been developed [11], [12]. Independent component analysis (ICA) is the most popular signal separation method, which separates the signal of a mixture into statistically independent signals. ICA is a blind source separation method and does not require prior knowledge for signal decomposition. ICA has been applied to electroencephalograms (EEGs) for removing blink and muscle artifacts [11] and facial images for face recognition [12]. Recently, ICA has been applied to NIR and MIR spectra to decompose their spectra into statistically independent spectra [13], [14]. ICA is introduced to an NIR spectrum to decompose pure spectra from the original spectrum of the mixture [13]. Independent component regression (ICR), a combination of ICA and MLR, has been applied to NIR spectra to quantitatively evaluate glucose concentrations from spectra of mixtures [14]. However, ICs decomposed by ICR are not guaranteed to be correlated with pure component properties, because ICR extracts features without prior knowledge [15]. Moreover, there is no clear standard to decide the number of ICs to be decomposed. Gustafsson [16] reported that a small training set decreased the quality of the estimates of loading vectors and prediction performance.

The objective of this paper is to accurately evaluate both ethanol and glucose concentrations in an ethanol and glucose mixed solution. It has been observed that absorption peaks of ethanol and glucose overlap, which can cause a reduction of the estimation performance. This paper proposes a spectral separation method using a single absorption band for accurate evaluation of ethanol and glucose concentrations. Because of the use of prior knowledge for spectral separation, our method is simple, does not require any parameter tuning, and relies on mathematical assumptions different from those 
of ICA and ICR. In the computer simulations, estimation error of the proposed method was compared with regression analysis (RA). From the simulation results, the estimation errors of ethanol and glucose concentrations decreased from $5.40 \pm 5.41 \%(\mathrm{v} / \mathrm{v})$ and $0.73 \pm 0.73 \mathrm{~g} / \mathrm{dl}$, respectively, using RA to $0.46 \pm 0.65 \%(\mathrm{v} / \mathrm{v})$ and $0.40 \pm 0.57 \mathrm{~g} / \mathrm{dl}$, respectively, using the proposed method.

\section{AttenuAted Total ReFlectAnCE (ATR) ONE-SHOT FOURIER TRANSFORM INFRARED (FT-IR) SPECTROSCOPY}

We herein propose attenuated total reflectance (ATR) one-shot Fourier transform infrared (FT-IR) spectroscopy. Fig. 1 shows a schematic representation of the proposed method. ATR measures sample spectra using evanescent light produced by a high refractive-index prism. ATR enables high performance measurements regardless of the thickness and concentration distribution of the sample. Moreover, measurement sensitivity can be improved by changing the prism shape to produce multiple reflections. However, the usual ATR used with FT-IR has a disadvantage that it is not suitable for the measurement of a volatile sample, such as alcohol beverages, because of the measurement time. However, our one-shot Fourier spectroscopy [17] requires a shorter measurement time. High performance ethanol and glucose measurements will be achieved by combining ATR one-shot FT-IR and the spectral separation method we propose in this paper.

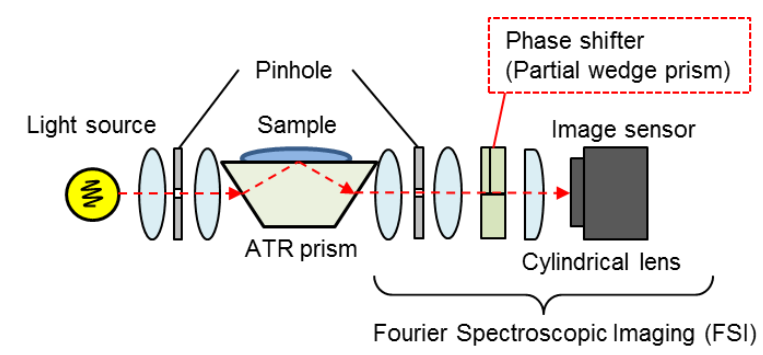

Fig. 1. Schematic representation of ATR one-shot FT-IR.

\section{PROPOSED METHOD}

\section{A. Outline}

We propose a spectral separation method using a single absorption band. In this paper, a mixed spectrum consisting of ethanol and glucose is separated into individual component spectra. Fig. 2 shows the flow of the proposed method. First, an absorption spectrum is obtained by measuring the sample and reference solutions by FT-IR. Second, we select a wavenumber that significantly reflects the glucose component, and robust with respect to ethanol component using regression analysis (RA). Any wavenumber is analytically evaluated based on the criteria for wavenumber selection, and the most adequate wavenumber is selected. Third, a "coefficient spectrum", which is used for ethanol and glucose spectral separation, is calculated based on the absorbance at the selected wavenumber. Fourth, the glucose spectrum in the mixed spectrum is estimated using the coefficient spectrum. The ethanol spectrum is obtained by subtracting the estimated glucose spectrum from the mixed spectrum. Finally, ethanol and glucose concentrations are evaluated using RA from the separated ethanol and glucose spectra.

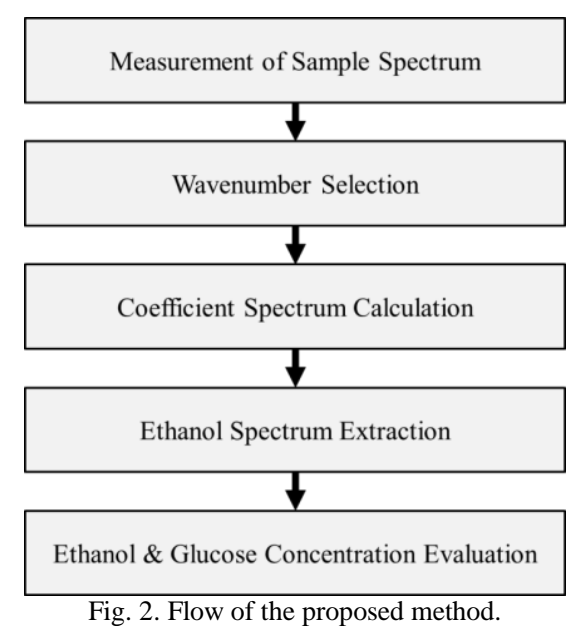

\section{B. Measurement of Sample Spectrum}

Three types of samples are prepared for spectral separation and the evaluation of ethanol and glucose concentrations. The absorption spectra are measured using FT-IR.

1) Samples for selecting a wavenumber that reflects the glucose component and is robust with respect to the ethanol component

Ethanol and glucose aqueous solutions are measured with different levels. The measured spectra are then used for wavenumber selection.

2) Samples for calculating the "coefficient spectrum" used for spectral separation

Glucose aqueous solutions are measured with the same level. The coefficient spectrum is then calculated using the measured glucose spectra and an absorbance at the selected wavenumber.

3) Samples for evaluating ethanol and glucose concentration from the mixed spectrum

Ethanol and glucose mixed aqueous solutions are measured with different levels. The ethanol and glucose concentrations are then evaluated by separating the measured spectrum into ethanol and glucose spectra.

\section{Wavenumber Selection}

The absorption peaks of ethanol and glucose overlap, as shown in Fig. 3. When we measure their mixed aqueous solution, the mixed spectrum of ethanol and glucose can be obtained. It is difficult to directly estimate each concentration from the mixed spectrum. To accurately evaluate the ethanol and glucose concentrations in the mixed aqueous solution, the mixed spectrum must be divided into ethanol and glucose spectra. In Fig. 3, a wavenumber, that is significant only for glucose can be observed. For example, 1110, 1024, 1267, 1285, 1292 and, $1467 \mathrm{~cm}^{-1}$ reflect only the glucose component. Our spectral separation method utilizes an absorbance at a wavenumber that is reflects glucose and is also robust with respect to the ethanol component. Any glucose spectrum can be reconstructed using a single absorption band if the proper wavenumber is selected (the reason for this will be explained later). By subtracting the reconstructed glucose spectrum from the mixed spectrum, the ethanol spectrum can be obtained. 


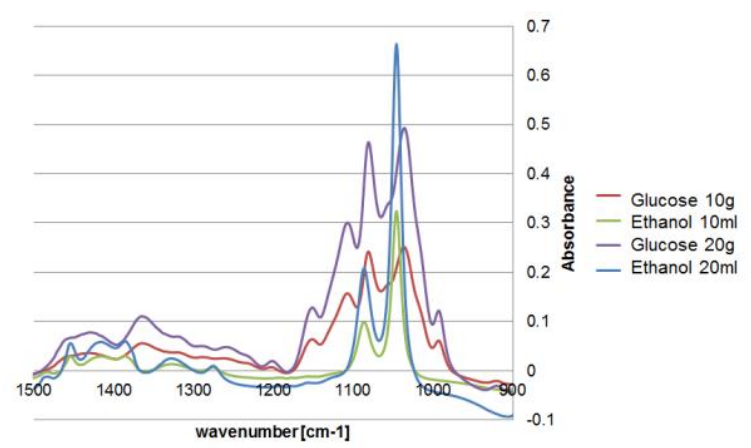

Fig. 3. Absorbance spectra of ethanol and glucose.

We select a wavenumber that is unique to glucose and robust with respect to ethanol. The relationship between the absorbance and the concentration is formulated using Lambert-Beer's law:

$$
A(\lambda)=\varepsilon(\lambda) c L
$$

where $A$ is an absorbance, $\lambda$ is a wavenumber, $\varepsilon$ is a molar absorption coefficient, $c$ is a sample concentration, and $L$ is the optical path length. It is assumed that $\varepsilon$ and $L$ are constant values and that the absorbance is linear with respect to the sample concentration. For instance, when the sample concentration doubles, the absorbance at any wavenumber also doubles. Therefore, the ratio of two absorbance at an arbitrary wavenumber is constant regardless of changes in the sample concentration. For this reason, any glucose spectrum can be reconstructed using a single absorption band if the appropriate wavenumber is selected. The relationship between a sample concentration and its absorbance is modeled using RA:

$$
\mathrm{y}=a_{1} x_{1}+a_{0}
$$

where $y$ is the sample concentration, $a$ is the regression coefficient, and $x$ is the sample absorbance. The best ideal wavenumber is selected by evaluating a goodness of a model based on the F-value, which is calculated using Eq. (3):

$$
\mathrm{F} \text { value }=\frac{(\hat{y}-\bar{y})^{2} / p}{(y-\hat{y})^{2} /(n-p-1)}
$$

where $n$ is the number of samples, $p$ is the number of variables, $y$ is a measured value, $\bar{y}$ is the average of $y$, and $\hat{y}$ is the estimated value using RA. The criteria for wavenumber selection are as follow:

- The model shows the highest F-value among all candidates.

- The correlation coefficient between the sample concentration and the absorbance at the selected wavenumber takes a positive value.

To select a wavenumber for spectral separation, we prepare ethanol and glucose solutions with different levels (referred to as A) in subsection "B. Measuring Sample Spectra"). These solutions are measured using FT-IR. The best wavenumber is selected by applying RA to the measured spectra. As the results show, $1024 \mathrm{~cm}^{-1}$ was selected as the ideal wavenumber, which is unique to the glucose component and robust with respect to the ethanol component.

\section{Coefficient Spectrum}

The ratio between two glucose absorbance at any wavenumber is constant according to Lambert-Beer's law shown in Eq. (1), regardless of changes to the sample concentration. Therefore, a glucose absorbance at any wavenumber can be reconstructed using an absorbance at the selected wavenumber of $1024 \mathrm{~cm}^{-1}$. Because the chosen wavenumber is unique to glucose and robust with respect to ethanol, we can calculate the ethanol spectrum from the mixed spectrum and the reconstructed glucose spectrum. We can also calculate the coefficient spectrum for glucose spectrum estimation from the mixed spectrum.

We prepare glucose solutions with the same levels (referred to as B) in subsection "B. Measuring Sample Spectra"). These solutions are measured using FT-IR. The average absorption spectrum is used to calculate the coefficient spectrum for glucose spectrum estimation. A coefficient spectrum is calculated using Eq. (4):

$$
a(\lambda)_{\mathrm{coe}}=\frac{A_{\mathrm{glu}}(\lambda)}{A_{\mathrm{glu}}(1024)}
$$

where $a_{\text {coe }}$ is a coefficient, $A_{\text {glu }}$ is the glucose absorbance, and $A_{\text {glu }}(1024)$ is the glucose absorbance at $1024 \mathrm{~cm}^{-1}$. By using this calculated coefficient spectrum (shown in Fig. 4), we can estimate the glucose spectrum from the mixed spectrum.

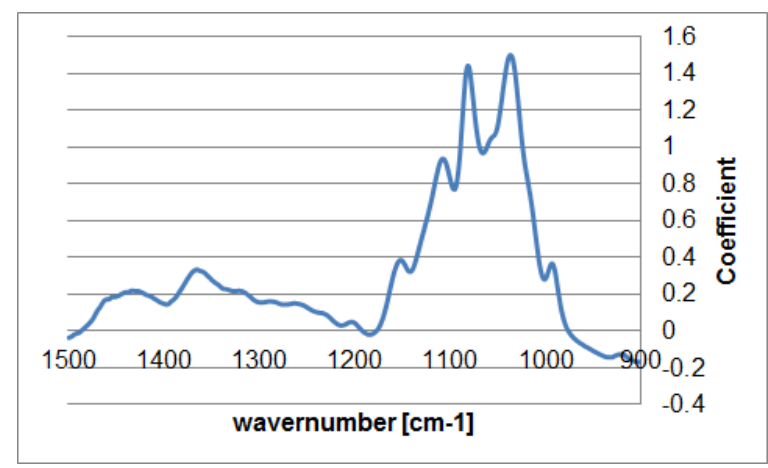

Fig. 4. A calculated coefficient spectrum.

\section{E. Ethanol Spectrum Extraction}

We calculate the ethanol spectrum using the coefficient spectrum calculated using Eq. (4). The ethanol spectrum is obtained by subtracting the reconstructed glucose spectrum from the mixed spectrum.

We prepare ethanol and glucose mixed solutions with different levels (referred to as C) in subsection "B.Measuring Sample Spectra"). These solutions are measured using FT-IR. The ethanol spectrum in the mixed spectrum is estimated using Eq. (5):

$$
A(\lambda)_{\text {etha }}=A(\lambda)_{\mathrm{mix}}-a(\lambda)_{\mathrm{coe}} A(1024)_{\mathrm{mix}}
$$

where $A_{\text {etha }}$ is the ethanol absorbance, $A_{\text {mix }}$ is the mixed absorbance, and $A(1024)_{\text {mix }}$ is the mixed absorbance at $1024 \mathrm{~cm}^{-1}$. The second term on the right-hand of the equation indicates the glucose absorbance. Fig. 5 shows a mixed spectrum and Fig. 6 shows a calculated ethanol spectrum. 


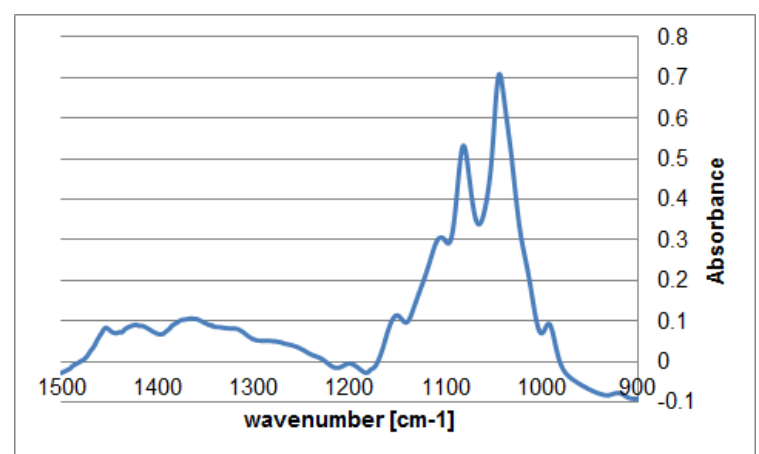

Fig. 5. A mixed absorbance spectrum.

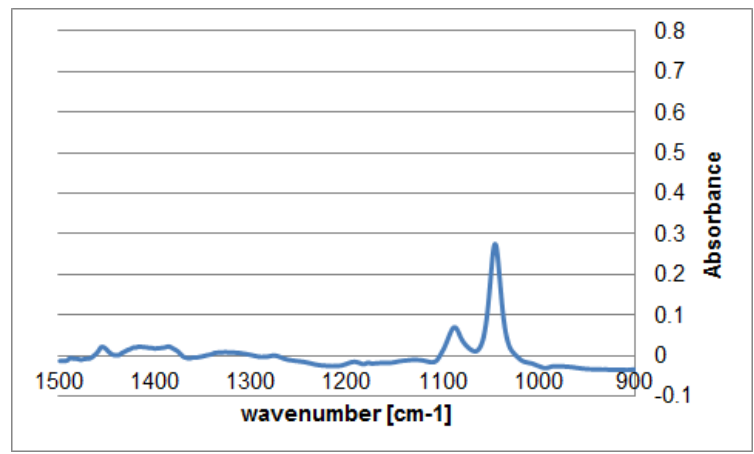

Fig. 6. Extracted ethanol absorbance spectrum.

\section{F. Ethanol and Glucose Concentration Evaluation}

The ethanol concentration in a mixed spectrum is calculated using the calculated ethanol spectrum. RA is used to calculate the ethanol concentration. On the other hand, the glucose concentration in the mixed aqueous solution is calculated using the reconstructed spectrum from the absorbance at $1024 \mathrm{~cm}^{-1}$.

\section{COMPUTER SIMUlations}

\section{A. Simulation Conditions}

The usefulness of the proposed method is evaluated by simulations. A sample spectrum is measured using FT-IR Spectrum Spotlight 200 developed by PerkinElmer Inc. with a measurement range of 4000 to $600 \mathrm{~cm}^{-1}$ and a resolution of 4 $\mathrm{cm}^{-1}$. A ZnSe liquid cell is used and the spacer size is $25 \mu \mathrm{m}$. To improve the $\mathrm{S} / \mathrm{N}$ ratio, a spectrum is accumulated 64 times. From the preliminary experiments, absorption spectra over the range of $1500 \sim 900 \mathrm{~cm}^{-1}$ were used for the simulations.

We compare the proposed method to the RA method. RA is directly applied to the mixed spectrum without spectral separation to estimate the ethanol and glucose concentrations. The estimation error is evaluated using the root mean square error (RMSE).

$$
\operatorname{RMSE}=\sqrt{\sum_{i=1}^{N} \frac{\left(y_{i}-\hat{y}_{i}\right)^{2}}{N}}
$$

where $N$ is the number of samples. This simulation was implemented on Microsoft Visual Studio 2010 on Windows 8. The program was generated using $\mathrm{C}$ language.

\section{B. Estimation Performance of Glucose Spectrum}

We evaluated the estimation error of the glucose spectrum. Fig. 7 shows the absorbance difference between the desired glucose spectrum and the estimated glucose spectrum. The absorbance difference was calculated using the following equation:

$E(\lambda)_{\mathrm{glu}}=\frac{1}{N} \sum_{i=1}^{N}\left|A_{i}(\lambda)_{\text {glu,desired }}-A_{i}(\lambda)_{\text {glu,estimated }}\right|$

where $A_{\text {glu,desired }}$ is the desired glucose spectrum and $A_{\text {glu,estimated }}$ is the estimated glucose spectrum by the proposed method. A greater absorbance differenceis observed in the range of $1140 \sim 1020 \mathrm{~cm}^{-1}$. An absorbance difference of 0.025 was found at $1083 \mathrm{~cm}^{-1}$, which correspond to a glucose concentration of $1.11 \mathrm{~g} / \mathrm{dl}$. In other words, if the absorbance at $1083 \mathrm{~cm}^{-1}$ was used to estimate the glucose concentration, the glucose concentration error would be $1.11 \mathrm{~g} / \mathrm{dl}$.

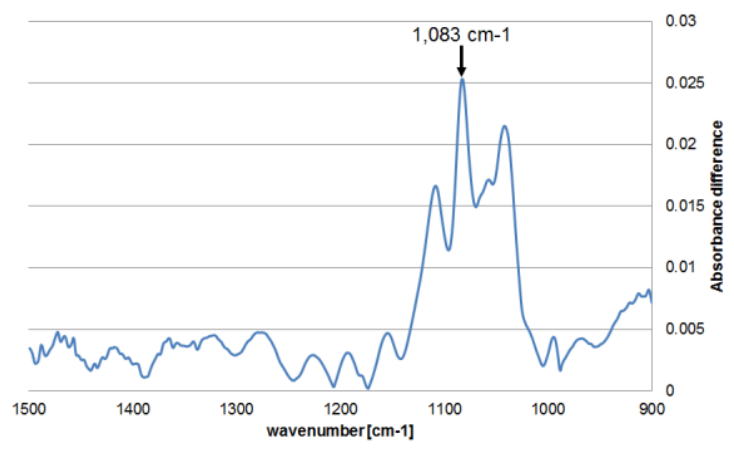

Fig. 7. An estimation error of glucose spectrum.

\section{Estimation Performance of Ethanol Spectrum}

We evaluated the estimation error of the ethanol spectrum. Fig. 8 shows the absorbance difference between the desired ethanol spectrum and the estimated ethanol spectrum. The absorbance difference was calculated using the following equation:

$E(\lambda)_{\text {eth }}=\frac{1}{N} \sum_{i=1}^{N}\left|A_{i}(\lambda)_{\text {eth,desired }}-A_{i}(\lambda)_{\text {eth,estimated }}\right|$

where $A_{\text {eth,desired }}$ is the desired ethanol spectrum and

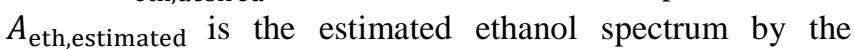
proposed method. An absorbance error is observed in the range of $1121 \sim 1031 \mathrm{~cm}^{-1}$. An absorbance difference of 0.024 was found at $1046 \mathrm{~cm}^{-1}$, which correspond to an ethanol concentration of $0.72 \%(\mathrm{v} / \mathrm{v})$. In other words, if the absorbance at $1046 \mathrm{~cm}^{-1}$ was used to estimate the ethanol concentration, the ethanol concentration error would be $0.72 \%(\mathrm{v} / \mathrm{v})$.

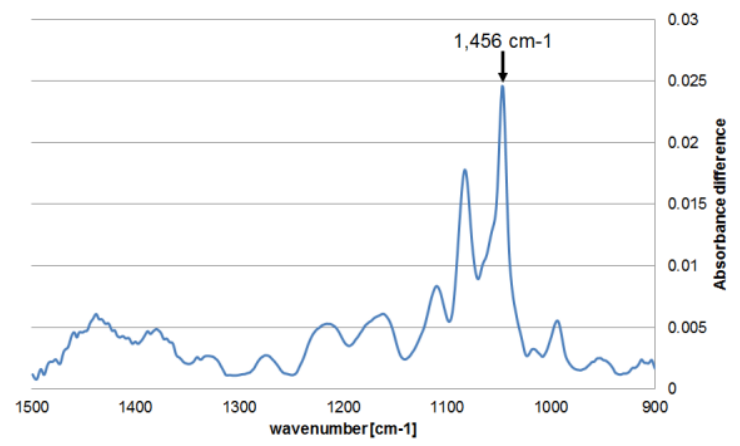

Fig. 8. An estimation error of ethanol spectrum.

\section{Ethanol and Glucose Concentration Evaluation}

The estimation errors of ethanol and glucose concentrations in a mixed aqueous solution are shown in 
Table I. The ethanol estimation errors are $0.46 \pm 0.65 \%(\mathrm{v} / \mathrm{v})$ and $5.40 \pm 5.41 \%(\mathrm{v} / \mathrm{v})$, and the glucose estimation errors are $0.40 \pm 0.57 \mathrm{~g} / \mathrm{dl}$ and $0.73 \pm 0.73 \mathrm{~g} / \mathrm{dl}$ using the proposed method and RA, respectively. The ethanol estimation error is drastically improved by proposed method compared with RA.
The selected wavenumbers are $1456 \mathrm{~cm}^{-1}$ and1047 $\mathrm{cm}^{-1}$ for ethanol evaluation, and $1275 \mathrm{~cm}^{-1}$ and $1112 \mathrm{~cm}^{-1}$ for glucose evaluation, determined using the proposed method and RA, respectively. The selected wavenumbers are varied depending on the methods.

TABLE I: AN ESTIMATION ERROR OF ETHANOL AND GLUCOSE CONCENTRATION

\begin{tabular}{ccccc}
\hline \hline & $\begin{array}{c}\text { Ethanol } \\
{[\%(\mathrm{v} / \mathrm{v})]}\end{array}$ & $\begin{array}{c}\text { Selected wavenumber } \\
\text { for ethanol }[\mathrm{cm}-1]\end{array}$ & $\begin{array}{c}\text { Glucose } \\
{[\mathrm{g} / \mathrm{dl}]}\end{array}$ & $\begin{array}{c}\text { Selected wavenumber } \\
\text { for glucose }[\mathrm{cm}-1]\end{array}$ \\
\hline Proposed method & $\mathbf{0 . 4 6} \pm \mathbf{0 . 6 5}$ & 1456 & $\mathbf{0 . 4 0} \pm \mathbf{0 . 5 7}$ & 1275 \\
RA & $5.40 \pm 5.41$ & 1047 & $0.73 \pm 0.73$ & 1112 \\
\hline \hline
\end{tabular}

\section{E. Discussions}

The proposed method showed a smaller RMSE than the RA method with respect to both ethanol and glucose concentrations. Because the proposed method separated the mixed spectrum into ethanol and glucose spectra, the effect of one component on the evaluation of the concentration of the other component was reduced. Moreover, RA resulted in a larger RMSE compared to the proposed method because the ethanol and glucose spectra overlapped, thus a suitable wavenumber for the concentration evaluation could not be determined. The selected wavenumber for ethanol and glucose evaluation was different between the proposed method and RA. The proposed method estimated the ethanol concentration using an absorbance at $1456 \mathrm{~cm}^{-1}$, whereas RA used an absorbance at $1047 \mathrm{~cm}^{-1}$. This variation resulted from the spectra separation used in the proposed method, which influenced to the results of the wavenumber selection. From the simulation results, the proposed method has been proven useful for separating ethanol and glucose spectra from a mixed spectrum, and improving the ethanol and glucose estimation performance.

To achieve an accurate concentration estimation coefficient spectrum for calculating the glucose absorbance spectrum, a precise model is required. In Fig. 7 and Fig. 8, a large absorbance difference is observed around the glucose and ethanol peaks. This error can be cause of the reduction of the concentration estimation performance. The error of absorbance at $1024 \mathrm{~cm}^{-1}$ compared to the model is $0.006 \pm$ 0.008 . If an absorbance at $1024 \mathrm{~cm}^{-1}$ was 0.014 , the estimation error of the ethanol and glucose concentrations would be $0.71 \%(\mathrm{v} / \mathrm{v})$ and $0.82 \mathrm{~g} / \mathrm{dl}$, when using absorbance at $1456 \mathrm{~cm}^{-1}$, and $1275 \mathrm{~cm}^{-1}$, respectively. Therefore, a baseline shift correction and calculation of the coefficient spectrum using multiple wavenumbers will aid in reducing this modeling error.

\section{CONCLUSION}

This paper proposed a spectral separation method using a single absorption band. The coefficient spectrum for spectral separation was calculated using the glucose absorbance at $1024 \mathrm{~cm}^{-1}$. The ethanol absorbance spectrum was estimated from the mixed absorbance spectrum using the coefficient spectrum. The ethanol concentration was estimated using the extracted ethanol absorbance spectrum. From the simulation results, the RMSEs of the proposed method were determined to be $0.46 \pm 0.65 \%(\mathrm{v} / \mathrm{v})$ and $0.40 \pm 0.57 \mathrm{~g} / \mathrm{dl}$ for ethanol and glucose concentrations, respectively. The RMSEs for RA were determined to be $5.40 \pm 5.41 \%(\mathrm{v} / \mathrm{v})$ and $0.73 \pm 0.73$ $\mathrm{g} / \mathrm{dl}$ for ethanol and glucose, respectively. We thus verified that the proposed method was useful for the evaluation of ethanol and glucose concentrations in a mixed absorption spectrum.

The signal separation performance needed to be improved to accurately estimate the concentration of the sample. The coefficient spectrum was calculated based solely on the absorbance at $1024 \mathrm{~cm}^{-1}$. To calculate a coefficient spectrum that is robust with respect to noise and measurement error, the absorbance at multiple wavenumbers may be helpful. This paper was limited to the evaluation using aqueous solutions. We will apply the proposed method to a measured spectrum of rice wine to evaluate its ethanol and glucose concentrations.

\section{ACKNOWLEDGMENT}

This work was supported by a Young Research Grant from Kagawa University in Japan.

\section{REFERENCES}

[1] A. Nordon, A. Mills, R. T. Burn, F. M. Cusick, and D. Littlejohn, "Comparison of non-invasive NIR and raman spectrometries for determination of alcohol content of spirits," Analytica Chimica Acta, vol. 548, no. 1, pp. 148-158, 2005.

[2] F. A. Iñón, S. Garrigues, and M. de la Guardia, "Combination of mid-and near-infrared spectroscopy for the determination of the quality properties of beers," Analytica Chimica Acta, vol. 571, no. 2, pp.167-174, 2006.

[3] D. W. Lachenmeier, "Rapid quality control of spirit drinks and beer using multivariate data analysis of fourier transform infrared spectra," Food Chemistry, vol. 101, no. 2, pp. 825-832, 2007.

[4] P. D. Martin, D. F. Malley, G. Manning, and L. Fuller, "Determination of soil organic carbon and nitrogen at the field level using near-infrared spectroscopy," Canadian Journal of Soil Science, vol. 82, no. 4, pp. 413-422, 2002.

[5] L. F. B. de Lira, M. S. de Albuquerque, J. G. A. Pacheco, T. M. Fonseca, E. H. D. S. Cavalcanti, L. Stragevitch, and M. F. Pimentel, "Infrared spectroscopy and multivariate calibration to monitor stability quality parameters of biodiesel," Microchemical Journal, vol. 96, no. 1, pp. 126-131, 2010.

[6] M. J. Martelo-Vidal and M. Vázquez, "Determination of Polyphenolic compounds of red wines by UV-VIS-NIR Spectroscopy and chemometrics tools," Food Chemistry, vol. 158, no. 1, pp. 8-34, 2014.

[7] K. Wójcicki, I. Khmelinskii, M. Sikorski, F. Caponio, V. M. Paradiso, C. Summo, and E. Sikorska, "Spectroscopic techniques and chemometrics in analysis of blends of extra virgin with refined and mild deodorized olive oils," European Journal of Lipid Science and Technology, pp.1-11, 2014.

[8] V. Di Egidio, N. Sinelli, G. Giovanelli, A. Moles, and E. Casiraghi, "NIR and MIR spectroscopy as rapid methods to monitor red wine fermentation," European Food Research and Technology, vol. 230, no. 6, pp. 947-955, 2010.

[9] F. Shen, Y.vYing, B. Li, Y. Zheng, and J. Hu, "Prediction of sugars and acids in chinese rice wine by mid-infrared spectroscopy," Food Research International, vol. 44, no. 5, pp. 1521-1527, 2011. 
[10] J. H. Jiang, R. J. Berry, H. W. Siesler, and Y. Ozaki, "Wavelength interval selection in multicomponent spectral analysis by moving window partial least-squares regression with applications to midinfrared and near-infrared spectroscopic data," Anal. Chem., vol. 74, no. 14 , pp. 3555-3565, 2002.

[11] T. P. Jung, S. Makeig, C. Humphries, T. W. Lee, M. J. Mckeown, V. Iragui, and T. J. Sejnowski, "Removing electroencephalographic artifacts by blind source separation," Psychophysiology, vol. 37, no. 2, pp. 163-178, 2000.

[12] M. S. Bartlett, J. R .Movellan, and T. J. Sejnowski, "Face recognition by independent component analysis," IEEE Transactions on Neural Networks, vol. 13, no. 6, pp. 1450-1464, 2002.

[13] J. Chen and X. Z. Wang, "A new approach to near-infrared spectral data analysis using independent component analysis," Journal of Chemical Information and Computer Sciences, vol. 41, no. 4, pp. 992-1001, 2001

[14] A. Al-Mbaideen and M. Benaissa, "Determination of glucose concentration from NIR spectra using independent component regression," Chemometrics and Intelligent Laboratory Systems, vol. 105, no. 1, pp. 131-135, 2011.

[15] C. Zhao, F. Gao, and F. Wang, "An improved independent component regression modeling and quantitative calibration procedure," AIChE journal, vol. 56, no. 6, pp. 1519-1535, 2010.

[16] M. G. Gustafsson, "Independent component analysis yields chemically interpretable latent variables in multivariate regression," Journal of Chemical Information and Modeling, vol. 45, no. 5, pp. 1244-1255, 2005.

[17] Y. Inoue, I. Ishimaru, T. Yasokawa, K. Ishizaki, M. Yoshida, M. Kondo and N. Tanaka, "Variable phase-contrast fluorescence spectrometry for fluorescently stained cells," Applied Physics Letters, vol. 89 , no. 12 , p. $121103,2006$.

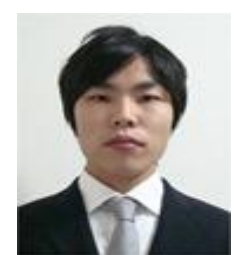

Satoru Suzuki received B.E. and M.E. degrees from Tokyo University of Agriculture and Technology in Japan in 2008 and 2009, respectively. He received a $\mathrm{Ph} . \mathrm{D}$. from Tokyo University of Agriculture and Technology in Japan in 2011. Since 2012, he has served as a postdoctoral fellow at Kagawa University in Japan. His major field of study is signal processing Dr. Suzuki is a member of The Institute of Electrical Engineers of Japan and Research Institute of Signal Processing Japan.

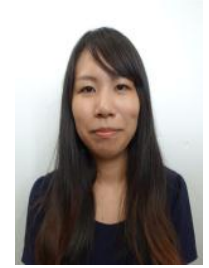

Satsuki Hosono is a fourth-year student of intelligent mechanical systems engineering, Faculty of Engineering, Kagawa University. Her interest is a component quantitative measurement of food and drink by spectroscopy using the mid-infrared light. Ms Hosono is a student member of The Japan Society for precision engineering and The Japan Society of Applied Physics.

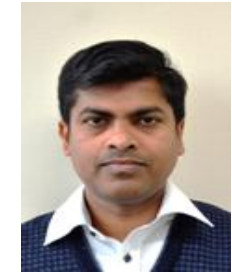

Pradeep K. W. Abeygunawardha received B.Sc. (Eng.) honours degree in electrical engineering from the University of Moratuwa and MSc and Ph.D degrees in robotic engineering from Keio University, Japan in 2006 and 2010respectively. He was a senior lecturer in Sri Lanka Institute of Information Technology (SLIIT) from 2010 to 2012. He served as the head of research center, SLIIT in 2011. Since 2012, He has been working as a postdoctoral researcher in Kagawa University, Takamatsu, Japan.

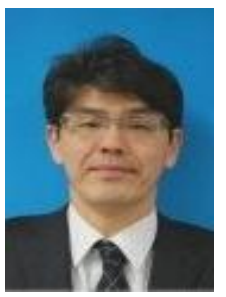

Kenji Wada is a professor of chemistry for medicine, Faculty of Medicine, Kagawa University, Japan. He received B.E., M.E., and $\mathrm{PhD}$ degrees from Kyoto University in 1987, 1989, and 1992, respectively, and spent his career at the Department of Hydrocarbon Chemistry, Kyoto University as an assistant professor, a senior lecturer, and an associate professor (1992-2013).His current research interests focus on the catalysis and the spectroscopy for diagnosis

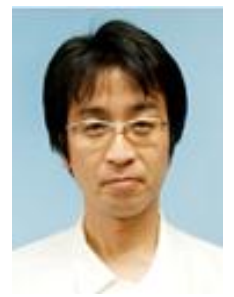

Akira Nishiyama received M.D. from Kagawa Medical University in Japan in 1993, and Ph.D. from Kagawa Medical University in Japan in 1999. He is a professor of the department of Pharmacology, Kagawa University Medical School in Japan. His research field is renal physiology, hypertention and kindney injury. Prof. Nishiyama is a member of The American Heart Association (Fellow), and The Society of Cardiovascular Endocrinology and Metabolism (Councilor).

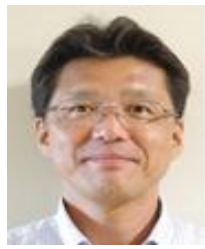

Ichiro Ishimaru received the B.S. degree in mechanical engineering from Osaka University, Japan, in 1987, and then the doctor of engineering from the University of Tokyo, Japan, in 1999. He joined Production Engineering Research Laboratory in Hitachi Corporation from 1987. He is a professor of the Department of Intelligent Mechanical Systems Engineering, Faculty of Engineering in Kagawa University from 2008. His main work is the optical biomedical measurement such as the spectroscopic tomography of bio-membrane. Prof. Ishimaru is a member of The Japan Society for Precision Engineering and The Optical Society of Japan. 
